\title{
Multi-Channel Neutron Emission and Triton Burn-up Measurement on JT-60U Using Digital Signal Processors
}

\author{
Keiichi ISHII, Kouji SHINOHARA ${ }^{1)}$, Masao ISHIKAWA ${ }^{1)}$, Toshio OKUJI ${ }^{2)}$, Mamoru BABA ${ }^{2)}$, \\ Mitsutaka ISOBE ${ }^{3)}$, Sumio KITAJIMA and Mamiko SASAO \\ Department of Quantum Science and Energy Engineering, Tohoku University, Sendai 980-8579, Japan \\ 1) Japan Atomic Energy Agency, 801-1 Mukoyama, Naka 311-0193, Japan \\ ${ }^{2)}$ Cyclotron and Radioisotope Center, Tohoku University, Sendai 980-8578, Japan \\ 3) National Institute for Fusion Science, 322-6 Oroshi-cho, Toki 509-5292, Japan
}

(Received 9 January 2009 / Accepted 8 July 2009)

\begin{abstract}
In JT-60U, the multi-channel neutron profile monitor measures the line-integrated neutron emission. A digital signal processing (DSP) system was applied to all neutron detectors in the neutron profile monitor. A new 2D mapping method for DSP data analysis was developed to discriminate neutrons from $\gamma$-rays. The combination of DSP and the 2D map enabled the simultaneous measurement of DD and DT neutrons with one detector. The time evolutions of DD and of DT neutrons showed different responses to the ITB formation, depending on the viewing chord.
\end{abstract}

(C) 2010 The Japan Society of Plasma Science and Nuclear Fusion Research

Keywords: JT-60U, neutron diagnostics, Digital signal Processors, triton burn-up

DOI: $10.1585 /$ pfr.5.S1043

\section{Introduction}

The information on the energetic ion behavior in deuteron heated deuterium plasma can be obtained by measuring the line-integrated neutron emission rate with neutron detectors viewing the plasma along a number of chords (neutron profile monitor) [1]. To avoid contamination by $\gamma$-rays and the scattering component in a neutron profile measurement, it is important to use a detector that can discriminate neutrons from $\gamma$-rays and is insensitive to low-energy neutrons. To discriminate neutrons from $\gamma$-rays, several types of scintillator, e.g., NE213, Stilben crystals, and NE451, have been used [1], combined with sophisticated discrimination electronics, which require a finite process time and hence prevent the high counting rate measurement.

To overcome this problem in the count rate a digital signal processing (DSP) system was developed and applied to all neutron detectors of the multi-channel neutron profile monitor of seven viewing chords in JT-60U [2-6]. It is important to develop rapid and reliable $n-\gamma$ discrimination logics and software for the digital data analysis.

In the present study, a new 2D n- $\gamma$ discrimination mapping method was introduced and applied to the DSP data from the neutron profile monitor of JT-60U. Using this new system, neutron profile measurements were successfully carried out during the 2008 campaign of JT-60U.

In JT-60U, DD neutrons were produced in a deuterium plasma heated by $\mathrm{D}^{0}$-beams. The DT neutrons were also produced by triton burn-up, and their time evolution can

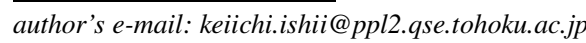

be used to predict $3.5 \mathrm{MeV} \alpha$-particle behavior in a fusion reactor. The separation of DT neutrons from DD neutrons was clearly performed, and the time evolutions of both DD and DT neutrons were obtained. They showed different behaviors depending on the viewing chord.

\section{Neutron Profile Monitor of JT-60U}

In JT-60U, the multi-channel neutron profile monitor had seven viewing chords: six chords view the plasma poloidally and one chord views vertically. Details of the viewing and collimation systems were described previously [2].

\subsection{Hardware}

Each viewing chord was equipped with a stilben crystal neutron detector. Stilben crystals are sensitive not only to neutrons but also to $\gamma$-rays. It is necessary to discriminate neutrons from $\gamma$-rays. An analog neutron- $\gamma$ pulse shape discrimination circuit had been used to measure collimated neutron flux [2]. However, the maximum count rate of the analog $n-\gamma$ discrimination circuit was limited below $\sim 1 \times 10^{5} \mathrm{cps}$. To overcome this problem in the count rate, a digital signal processing (DSP) system was developed for neutron detectors $[3,5]$. In this DSP system, output pulses from the anode of a photo multiplier tube (PMT) of a detector are recorded as a continuous waveform using a fast flash analog-to-digital converter (flash ADC) [6]. One pulse decayed typically within $\sim 100 \mathrm{~ns}$. Therefore, the flash ADC was required with the nanosecond range sampling rate of digitizing output. 
This DSP system had been extended to seven chords of the neutron collimator system [6]. The recorded data size was $7 \mathrm{~GB}$ per discharge. The sampling rate was usually $200 \mathrm{M}$-samples/s, which was chosen from the optimization between the pulse shape discrimination quality and the time period covered by the system, although the maximum sampling rate was $5 \mathrm{G}$-samples/s $[5,6]$. With the ADC sampling rate of $200 \mathrm{M}$-samples/s, the duration covered by the data acquisition scheme was about $2.68 \mathrm{~s}$. Recorded data were stored once in the flash ADC, and transferred onto a PC after measurement.

Recorded data were analyzed using off-line software (1) to discriminate neutrons from $\gamma$-rays, (2) to obtain a pulse height spectrum to separate DD and DT neutrons in every time bin, typically $10 \mathrm{~ms}$, and (3) to determine the time evolution for both DD and DT neutrons.

\subsection{Pulse shape analysis}

To discriminate neutrons from $\gamma$-rays, a charge integration method was used in the software, based on the difference in decay time of an output pulse. This system successfully achieved a counting rate capability higher than $\sim 1 \times 10^{5}$ counts/s [5]. The software for pulse shape analysis had the following functions: (1) determines the maximum of the digitized pulse height for each pulse, and saves it as "height," (2) produces 3 integrals, i.e., "fast," "slow," and "total," and (3) rejects pile up events Neutron- $\gamma$-ray $(\mathrm{n}-\gamma)$ discrimination was performed using the charge integration method in which each pulse was integrated for two time intervals as shown in the software functions. The integral for the time interval near the pulse peak was defined as "fast," while the other was called "slow." The value of "total" was integrated from the time when the pulse rose up to the end of the slow interval.

Figure 1 shows the pulse shape digitized in $2 \mathrm{G}$ samples/s, and fast and slow time intervals. As shown in Fig. 1, the decay time of neutrons is longer than that of $\gamma$-rays. That is, the charges of neutrons are lager than those of $\gamma$-rays, which have the same pulse height as neutrons. Using three values for one pulse, "fast," "slow," and "height," a two-dimensional (2D) map in height-slow normalized with (fast+slow) space can be plotted to discriminate neutrons (Fig. 2). The color scale on the right hand side indicates the number of events in one mesh on the $400 \times 400$-meshed 2D map of the pulse height and the slow/(fast+slow) ratio. Neutrons were discriminated graphically using this 2D map.

In Fig. 2, there are two normalized slow peaks at $\sim 0.15$ and $\sim 0.25$. The peak with a small charge is due to $\gamma$ rays. Most of the pulses due to neutrons are distributed in the low height region below the height of 0.2. Pulses distributed in this region are considered to be DD neutrons. A few neutrons distributed above the height of 0.2 can be DT neutrons, which are produced in the DT fusion reaction emitting at $14 \mathrm{MeV}$. In Fig. 2, neutron events are not sep-
E049813 2 G-samples/s

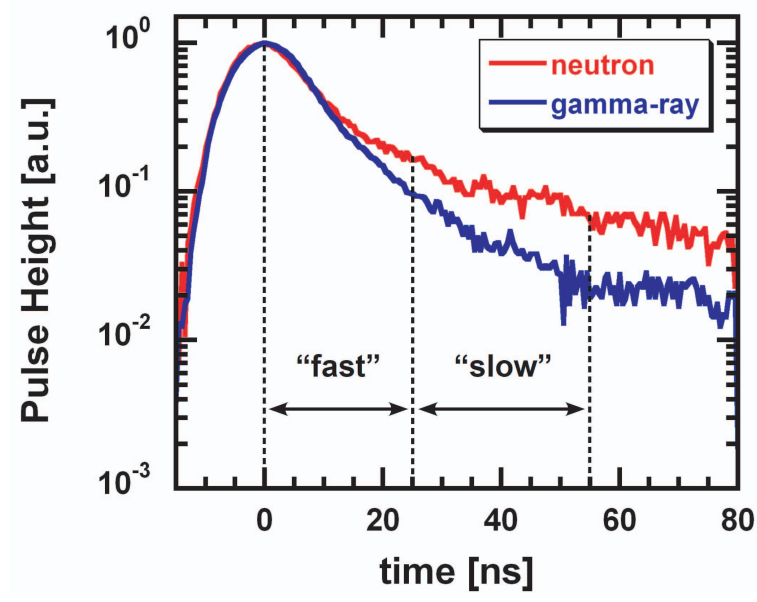

Fig. 1 An example of a pulse shape digitized with 2 Gsamples/s. Time intervals of fast and slow are indicated by dotted lines. The decay time of neutrons is longer than that of $\gamma$-rays.

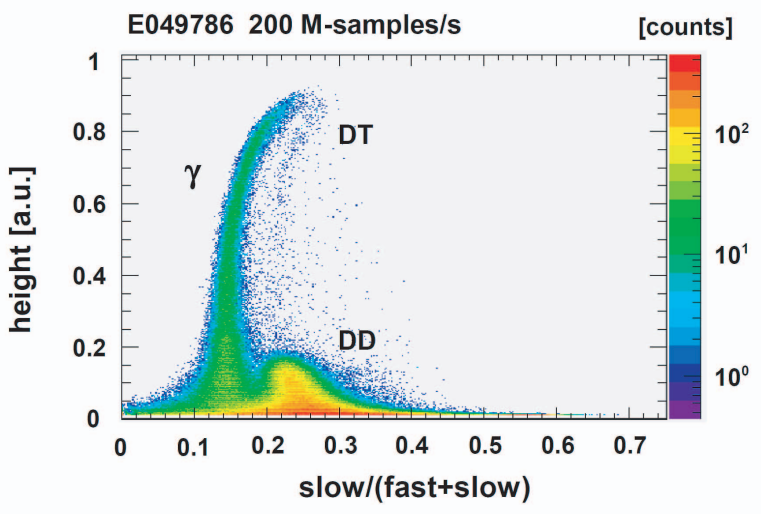

Fig. 2 A 2D map in height-slow space for $\mathrm{n}-\gamma$ discrimination.

arated from those of $\gamma$-rays in the low energy region with a height below 0.05 , and in the high-energy region above 0.7. To resolve the problem in the low energy region, the value of "total," which was the integration over the whole pulse, was used. fast/total and slow/total were used as the parameters of the 2D map shown in Fig. 3. The color scale is used to indicate the number of events in a mesh on the $400 \times 400$-meshed 2D map.

There are two groups in Fig. 3, and neutrons can be discriminated clearly from $\gamma$-rays by comparing with Fig. 2. To investigate the features of a new $n-\gamma$ giscrimination method, we selected the neutron region in Fig. 3 and plotted these pulses again in the conventional 2D map with parameters height and slow/(fast+slow). The separation was successful for low energies. However, some high-energy $\gamma$-rays were counted as neutrons. Therefore, a conventional height-slow space discrimination was further performed for neutron events selected by the new 2D map of the normalized fast-slow space (double 2D discrimina- 


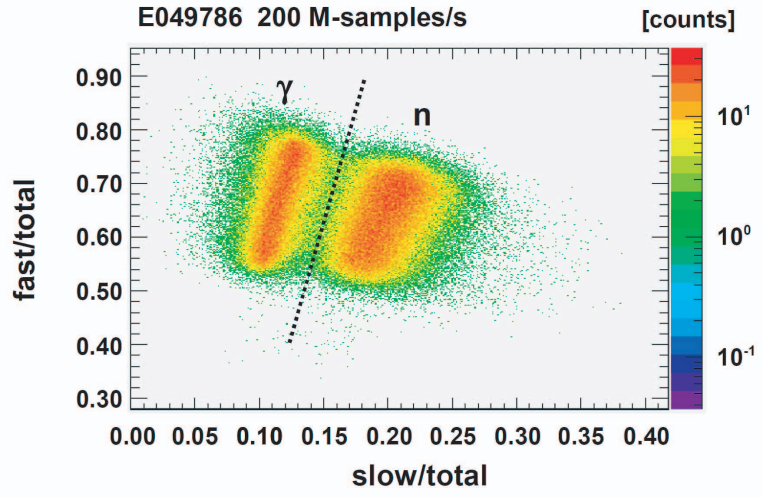

Fig. 3 A new 2D map in fast-slow space normalized with total. Neutrons are discriminated from $\gamma$-rays clearly.

tion).

To obtain the profile of DT neutron emission rate, it is important to discriminate DT neutrons for all detectors. The time interval of the charge integration method was the same value for all detectors on the supposition that all detectors had the same pulse shape. However, some detectors did not discriminate DT neutrons from energetic $\gamma$ rays. To resolve this problem, pulse shape analysis was performed using pulses recorded at a high sampling rate (2 G-samples/s). The results indicated that pulse shape was different for each detector. The time intervals were changed for each detector in the software, and then all detectors successfully discriminated DT neutrons from energetic $\gamma$-rays.

\section{Triton Burn-up}

In JT-60U, DD neutrons were measured mainly because energetic deuterium was injected as a neutral beam (NB) into the deuterium plasma. Not only $2.5 \mathrm{MeV}$ neutrons but also $1 \mathrm{MeV}$ tritons are produced through the DD fusion reaction. When tritons are confined in the bulk plasma and slow down to the energy region suitable for DT reaction by collision mainly with electrons, they may react with deuterons and then produce $14.1 \mathrm{MeV}$ neutrons. In the DSP system, DT neutrons were measured as well as DD neutrons simultaneously using the same detector. Figure 4 shows the pulse height distribution of neutrons discriminated from $\gamma$-rays using the double 2D discrimination. In Fig. 4, the edge near a height of 0.15 is considered the DD neutron edge. The counts distributed above 0.15 are DT neutrons, indicating that the DSP system successfully achieves DT neutron detection. High neutron counts in the low-energy region are considered to be scattered neutron components.

To determine the time evolutions of DD and DT neutrons, two regions were defined in the height distribution. As shown in Fig. 4, one region defined for DD neutrons was from 0.05 to 0.15 , while the region for DT neutrons was above 0.4. A histogram of neutrons in the each re-

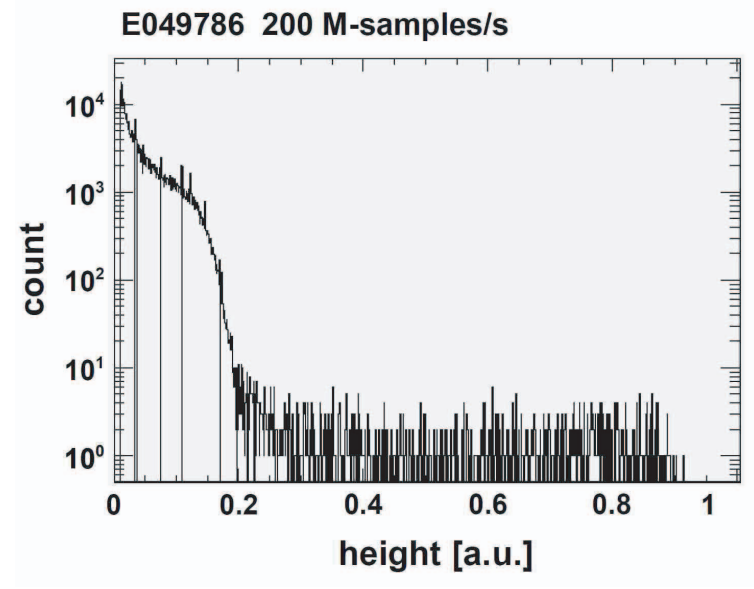

Fig. 4 The height distribution of neutrons discriminated from $\gamma$ rays. In the DSP system, DT neutrons are measured as well as DD neutrons simultaneously using the same detector.

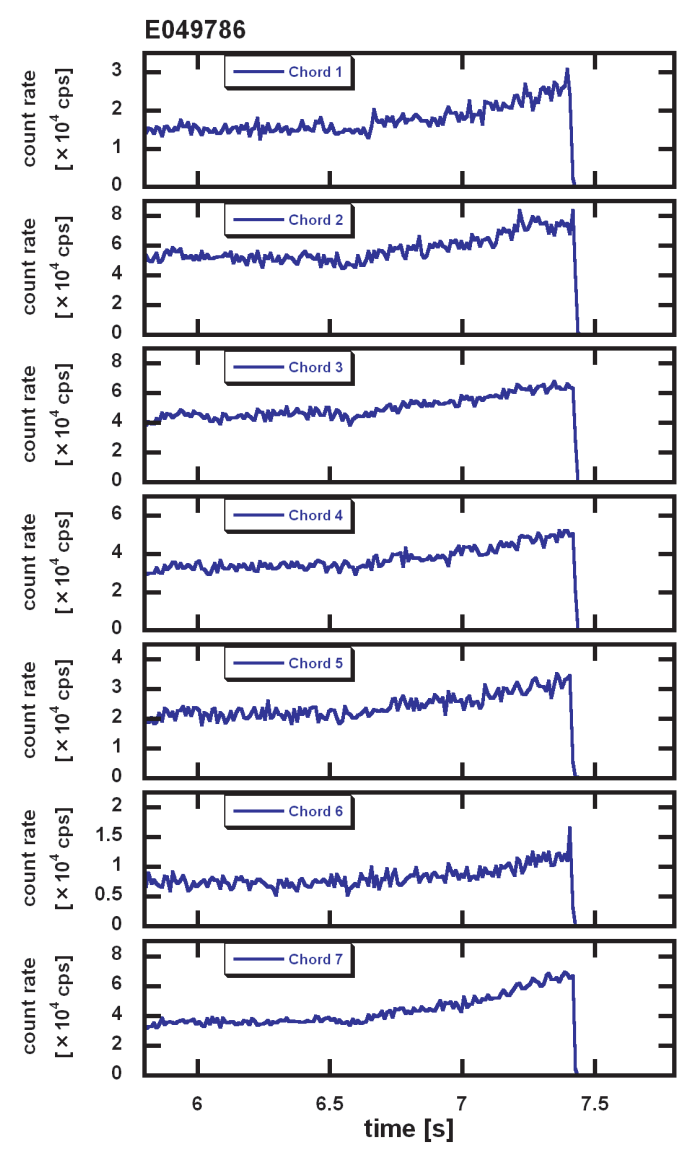

Fig. 5 The time evolution of the DD neutron emission rate measured by DSP system.

gion was produced against time, and the time distribution of neutrons was obtained. Figure 5 and Fig. 6 show the time evolution of the DD neutron emission rate and DT neutron emission rate measured by DSP system respectively. The DT neutron emission rate was less than that 


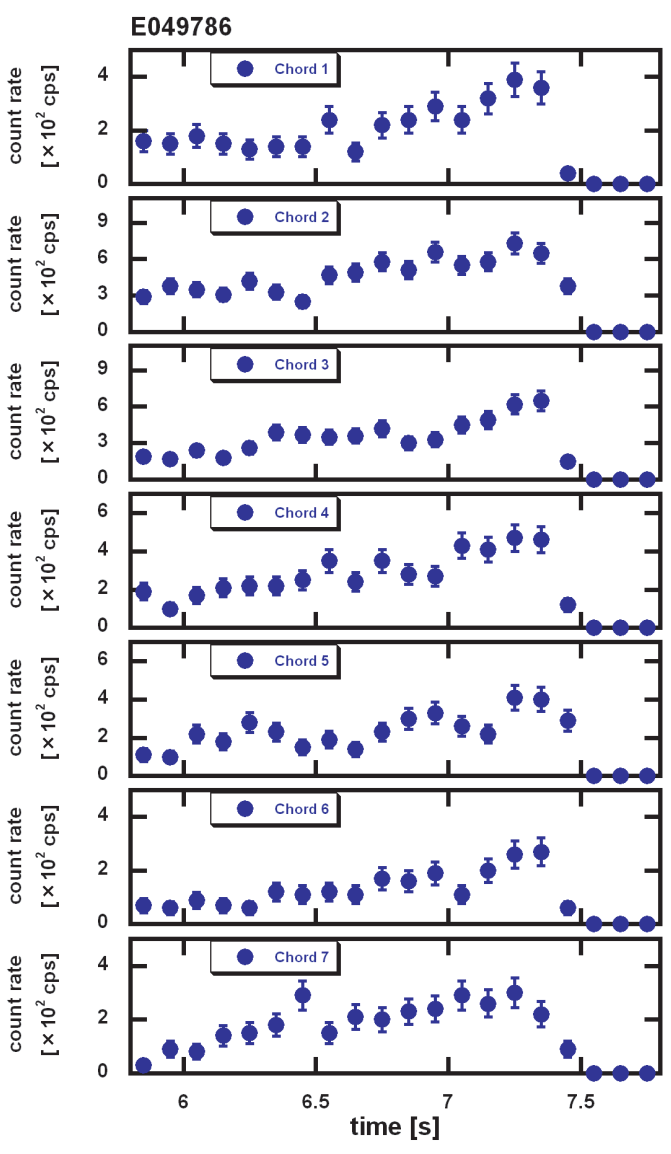

Fig. 6 The time evolution of the DT neutron emission rate measured by DSP system. The simultaneous measurement of DD and DT neutrons with one detector is succeeded.

of DD neutrons by the order of 2-3 in Fig. 4, similar to the ratio reported from other large tokamaks [1]. Therefore, it is necessary for the counting time interval of DT neutrons to be longer than that of DD neutrons to increase statistical precision. In Fig. 6, the time interval for DT neutrons is ten times longer than that for DD neutrons. Therefore, the time evolution of DT neutron is shown with dots due to low time resolution. If the DT neutron emission rate is higher, the time resolution can be higher.

Figure 7 shows the time evolution of neutron emission rate measured by the DSP system and plasma parameters. In this discharge, the plasma was heated by the $13 \mathrm{MW}$ positive ion-based neutral beam (PNBI) and $\sim 2.9 \mathrm{MW}$ electron cyclotron heating $(\mathrm{ECH})$. The plasma current was 1.2 MA and the toroidal magnetic field was 3.7 T. The plasma was disrupted at $7.4 \mathrm{~s}$.

The DSP system measured the line-integrated neutron emissivity, not local neutron emissivity. The absolute value of the line-integrated neutron emission rate in a chord cannot be compared with those in other chords because the geometrical efficiency and the detection efficiency have not yet been considered. During measurement in the DSP system, the power of PNB was almost constant and ECH was

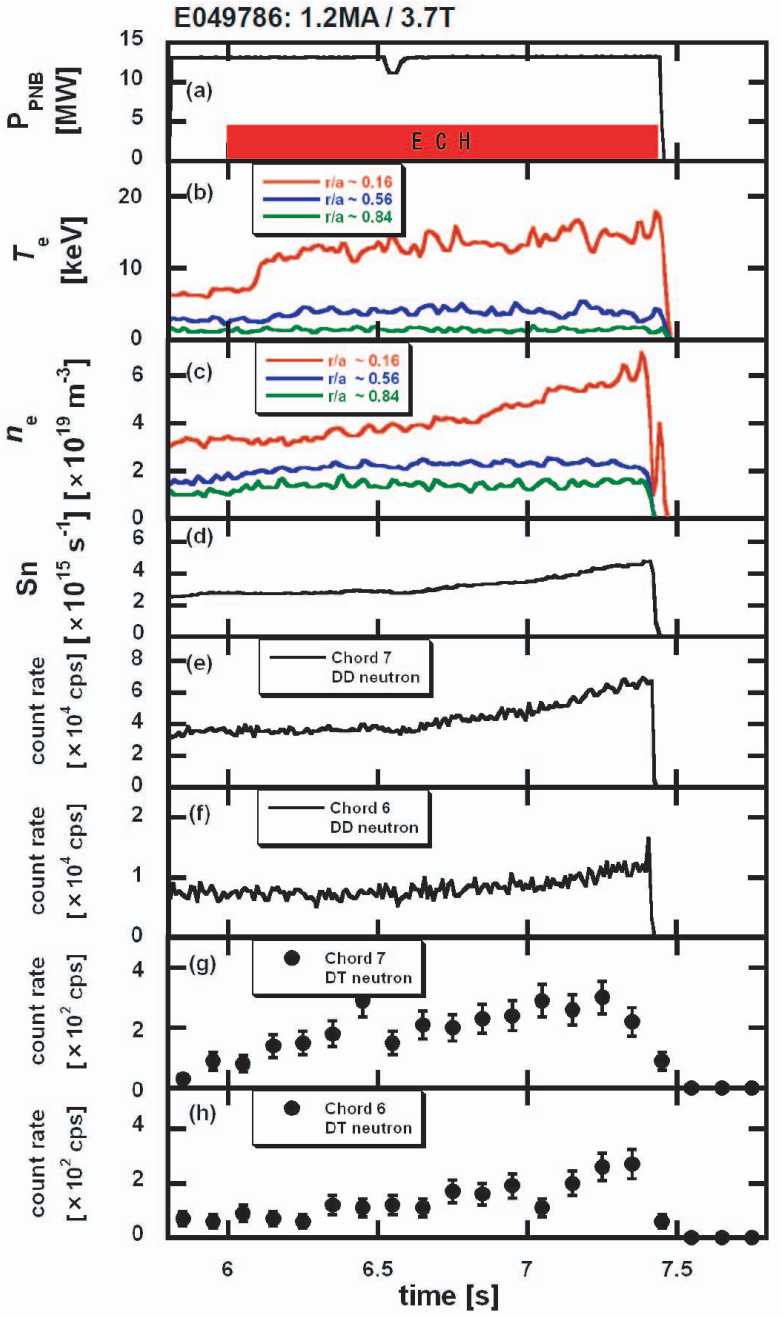

Fig. 7 The time evolution of: (a) input power of PNB and ECH, (b) electron temperature, (c) electron density, (d) total neutron emission rate measured with a fission chamber, (e) count rate of DD neutron in chord 7, which views the core region of the plasma, (f) count rate of DD neutrons in chord 6 viewing the edge region, $(\mathrm{g})$ count rate of DT neutrons in chord 7 , and $(\mathrm{h})$ count rate of DT neutrons in chord 6.

injected from $6.0 \mathrm{~s}$. Electron temperature and density increased remarkably from $6.3 \mathrm{~s}$ in the core region. That is, an internal transport barrier (ITB) was formed. Total neutron emission rate measured by the fission chamber increased gradually from $6.5 \mathrm{~s}$ until disruption. The time evolution of DD neutrons measured by the DSP system was similar to that of a fission chamber. However, the DD neutron emission rate in the central chord (chord 7) increased to a greater extent than that in the peripheral chord (chord 6) after ITB formation. The DD neutron emission is considered to reflect the density almost linearly because the dominant DD fusion component was not the thermal but that of the beam-plasma interaction and the input power of PNB was almost constant.

The DT neutron emission showed different time evo- 
lution in each chord. In chord 7, the count rate of DT increased by more than a factor of 3 after ITB formation. The DT emission rate in every chord changed time to time more the statistics, and was less steady than the DD emission. In general, the time response of DT neutrons is delayed by the slowing down time (typically over $1 \mathrm{~s}$ in the present case), and therefore it is affected by changes in diffusion, MHD instability, stochasticity, etc. [7]. In the present case, the change of classical diffusion can be excluded because the typical deflection time is longer than $1 \mathrm{~s}$. Further analyses of discharges and the study of the relations with various parameters are needed.

\section{Summary}

A digital signal processing (DSP) system was applied to every neutron detector of the neutron profile monitor of JT-60U. A double $n-\gamma$ discrimination mapping method of DSP data analysis was developed, where a new 2D map in a fast-slow space normalized with total was introduced. Using this system, DT neutrons were measured as well as DD neutrons in the DD plasma simultaneously with the same detector. The time evolution of DT neutrons was obtained for all chords.

DD neutron emission rate in the central chord increased to a greater extent than that in the chord view- ing the edge region after the appearance of the ITB. The time evolution of DT neutrons showed differences for each chord, which could not be explained by classical energetic ion diffusion.

\section{Acknowledgments}

The authors would like to express their appreciation to the JT-60U team for their contribution to the operation of JT-60U. This work was performed partially with the support of a Grant-in-Aid from the Ministry of Education, Culture, Sports, Science, and Technology of Japan, "Priority area of Advanced Burning Plasma Diagnostics."

[1] M. Sasao et al., Fusion Sci. Tech. 53, 604 (2008).

[2] M. Ishikawa, T. Nishitani, A. Morioka, M. Takechi et al., Rev. Sci. Instrum. 73, 4237 (2002).

[3] M. Ishikawa, T. Itoga, T. Okuji, M. Nakhostin, K. Shinohara et al., Rev. Sci. Instrum. 77, 10E706 (2006).

[4] M. Ishikawa, M. Takechi, K. Shinohara, C. Z. Cheng et al., Nucl. Fusion 47, 849 (2007).

[5] T. Itoga, M. Ishikawa, M. Baba, T. Okuji, T. Onishi et al., Radiat. Prot. Dosimetry 126, 380 (2007).

[6] K. Shinohara, T. Okuji, M. Ishikawa, M. Baba and T. Itoga, Rev. Sci. Instrum. 79, 10E509 (2008).

[7] W.W. Heidbrink and G.J. Sadler, Nucl. Fusion 34, 535 (1994). 